\title{
Interculturalidad y formación en Ciencias de la Salud
}

\section{Interculturalidad y formación en ciencias \\ de la salud \\ M. Chaib Akhdim}

En primer lugar quisiera agradecer la invitación que me han hecho para participar en este tercer encuentro de Profesores de Ciencias de la Salud. Creo que además ha sido todo un acierto por parte de los organizadores en considerar como uno de los temas a debatir la Interculturalidad y la formación en Ciencias de la Salud, puesto que a nadie se le escapa la importancia que hoy tiene para Catalunya, España y Europa la diversidad cultural y el fenómeno migratorio en nuestras sociedades. Es aquí donde la salud y el campo médico es un ámbito obligatorio de relación intercultural

Los movimientos migratorios y la educación tienen cada vez mayor protagonismo en el debate político europeo. La formación como factor educativo es requerida en diversos ámbitos, sin duda alguna en el de Salud.

Gestionar la inmigración en Ciencias de la Salud significa:

1 - atender a los estudiantes universitarios llegados de otros países respetando la diversidad cultural. En este sentido la Asociación ADMES presentó en el Forum de les Culturas 2004 una comunicación muy interesante e innovadora bajo el título de "Integración de los estudiantes de diferentes culturas a los estudios universitarios: punto de vista cultural y lingüístico"

2- Incorporar el elemento de la diversidad cultural en los centros sanitarios (hospitales, clínicas etc.) de forma que haya una mayor comunicación entre los profesionales de la salud y los pacientes de población extranjera con el fin de garantizar una atención sanitaria de cualidad. Es en este segundo punto en que basaré mi intervención.

La asistencia sanitaria en Cataluña es competencia exclusiva de la Generalitat, mientras que los ayuntamientos tienen competencias en los servicios y programas relacionados con la salubridad y la higiene. Unos y otros intentan garantizar el acceso de los inmigrantes extracomunitarios al sistema de salud y dar respuesta a sus problemas de salud específicos.

Los inmigrantes acuden a los centros sanitarios en muchas ocasiones con pocos conocimientos lingüísticos y con temor a no saber por una parte si van a ser comprendidos en su enfermedad o en sus dolencias por el profesional y por otra si van a ser capaces de transmitir lo que realmente les sucede. Por tanto ya se produce una barrera idiomática que dificulta la comunicación. Además del idioma se producen diferencias culturales que el profesional debe comprender para ofrecer una atención sanitaria correcta al paciente. Debe producirse un vínculo que permita la continuidad asistencial, un seguimiento del tratamiento, la instauración de pautas preventivas, tener en cuenta el proceso de adaptación del inmigrante al nuevo entorno con unas normas sociales y culturales diferentes de las propias etc. Sin duda alguna supone todo un reto tanto para los profesionales como para la población extranjera.

Dada la presencia creciente en Cataluña de personas llegadas de otras culturas, el mundo de la salud está continuamente reflexionando sobre como llegar a estas personas, por ello una de las soluciones es la contratación de mediadores interculturales de salud. Son personas residentes en el país que en un momento determinado pueden ser requeridos para ejercer de interlocutor entre el médico y el paciente. La mediación intercultural es un fenómeno bastante reciente. No existe unanimidad ni en el mundo científico ni sobre el terreno en lo que debería ser la mediación intercultural. Personalmente considero que la figura del mediador intercultural puede ser de gran utilidad si se le definen bien sus tareas y si está formado para llevara a cabo los objetivos que se esperan de él. En países europeos con más tradición migratoria tampoco lo tienen resuelto y sigue siendo un tema de debate. La definición de su función ha sido ampliamente debatida con los profesionales del sector y con los propios mediadores.

¿Debe actuar como intérprete exclusivamente o debe ir más allá ayudando a explicar la cultura del hospital y del médico al paciente, y el mundo del paciente al médico? En mi opinión debe hacer las dos cosas pero además creo que su figura debe ser un instrumento al que acudir cuando existen situaciones muy complicas sanitariamente, y es necesario buscar recursos institucionales en algunos casos y familiares o amistades en otros, puesto que existen momentos de gran tensión en hospitales porque no se sabe como solucionar ciertos problemas.

Bien es cierto que no se debe dejar en sus manos aquéllos aspectos que no le incumben y que no está preparado para ello, es decir que son tareas que corresponden a los profesionales. Por eso es enormemente importante definir las tareas que debe desarrollar.

Las relaciones culturales se producen en muchos lugares del ámbito sanitario como ocurre en las Oficinas de Farmacia, donde creo que es importante que los profesionales farmacéuticos se formen para garantizar una atención farmacéutica de calidad.

Los próximos años son de una gran trascendencia en el ámbito de la salud, aumentará el número de estudiantes de otras culturas hacia las universidades catalanas y españolas dado que existe cada vez más un mayor acercamiento y conocimiento de Cataluña y de España. Así mismo se producirá con el nuevo reglamento de extranjería una normalización de gran cantidad de personas que actualmente trabajan en nuestro país y que no poseen un permiso de residencia y trabajo. Una vez estabilizadas estas personas procederán a reagrupar a sus familias lo cual redundará sin duda alguna en la gestión de la Salud. Hemos de estar preparados para atender esta demanda no exenta de dificultades pero que supone 
una normalidad y una oportunidad de riqueza cultural puesto que estas personas no llegan vacías sino con un enorme bagaje cultural que debemos saber aprovechar e incorporar.

\section{Bioética intercultural para la salud global M.J. Buxó Rey}

Observatorio de Bioética y Derecho. UB.

Hoy las enfermedades no tienen fronteras, por lo tanto no pueden ser concebidas en términos locales, sino como problemas conectados con la pobreza y la marginalidad en todos los países del mundo. En su artículo sobre "El Estado, la Sociedad, los Derechos Humanos y la Salud: Desafíos éticos en el desarrollo de nuevas intervenciones", Solomon Benatar (Revista de Bioètica i Dret, 1, 2004) propone pensar la salud en términos globales.

Esta consideración de la salud global e integral obliga, por una parte, a detectar los impedimentos que dificultan crear condiciones de salud mundial y, por otra parte, buscar las vías y diseñar las estrategias necesarias para superar los obstáculos y alcanzar la cotas de salud que se entiende constituyen la calidad de vida.

En otras partes del mundo, y cada vez más en Occidente, la pobreza es cada vez más pobre, la salud y la extensión de las enfermedades infecciosas, HIV/sida, no decrece y, además, se agravan por las carencias alimentarias. Son así factores constantes la desnutrición, la falta y el acceso restringido al agua, la degradación ecológica, las diásporas intermitentes, y la privación de fármacos e instalaciones sanitarias adecuadas. La pobreza impide dar respuestas locales y desarrollar estrategias in situ para luchar contra la enfermedad, el incremento de la mortalidad infantil y la decreciente esperanza de vida.

Este es el diagnóstico de la situación, ahora bien, ¿qué tipo de respuesta dan las naciones a estas situaciones y qué calidad y probabilidades de éxito tienen para resolver estos problemas?

Se otorgan ayudas pero estas no llegan de forma unitaria y como retos para el desarrollo y la adquisición de competencias locales. La pluralidad de finalidades y la fragmentación de intereses de las naciones donantes no facilitan la coordinación y más bien, todo lo contrario, incrementan la complejidad organizativa y el entretenimiento burocrático de los países receptores.

Como propuesta de lo que hay que hacer, pienso que todos estaríamos de acuerdo en que hay que evitar acusaciones y el secuestro de la experiencia de los Otros pensando que sólo Occidente puede dar respuesta a los problemas. Así, un primer correctivo sería buscar vías para hacer propuestas multilaterales y orientar la investigación a objetivos de salud comunes. Aprender a entender conjuntamente qué se concibe por desarrollo, y cómo distribuir deberes y obligaciones.

En este sentido, es importante tener en cuenta quien y desde que instituciones se definen los problemas de salud. Y, sin duda, para definir los problemas tienen que incorporarse los propietarios del problema, o quien los sufre. En caso contrario no sólo se produce un secuestro de experiencias culturales, sino que difícilmente se resuelven los problemas porque la gente no se siente implicada, no entiende, no colabora y de ello resultan más efectos colaterales que estrategias reguladoras para aprender a vivir con los problemas.

Conviene recoger con técnicas etnográficas las formulas de desarrollo y las intervenciones a las que los países optan según sus necesidades pero también requerimientos culturales -sean restricciones religiosas, conocimientos tradicionales-. Sin duda las creencias religiosas juegan un papel fundamental en la aceptabilidad de las innovaciones, y de acuerdo con esos principios no todos los grupos culturales definen y aceptan los cambios en las prácticas sanitarias y alimentarias de la misma manera. La primera responsabilidad es la supervivencia, que no quiere decir ni conservadurismo ni inmovilismo, sino mantener la integridad del sistema ecológico y cultural.

$\mathrm{Y}$, en este punto, es importante insistir en la relevancia de la medicina tradicional. La etnomedicina no sólo aporta saberes a otros sistemas terapéuticos mediante la etnobotánica y la fitoterapia, sino también sobre la eficacia psicológica de las prácticas rituales, los efectos de placebo, que aportan seguridad al paciente y bienestar físico y psíquico. No hay que desestimar tampoco el carácter dinámico de las prácticas tradicionales ya que en contacto adoptan con facilidad al uso de medicamentos considerados en su doble perfil de útiles y prestigiosos. Es necesario, pues, estudiar estas combinatorias de sabiduría tradicional e inteligencia científica para evitar el descontrol y, a la vez, sincronizar vías de resolución terapéutica.

Y, por último, atendiendo al hecho que las enfermedades están vinculadas a la pobreza y a la exclusión social, la evaluación de los proyectos debería realizarse y ser auditados por equipos interdisciplinarios e internacionales que tengan en cuenta todos los factores socioculturales. Aclarar prioridades requiere evitar evaluaciones a gran escala basadas en estadísticas generales, y segregar los datos procedentes de trabajos de campo sistemáticos para saber donde incidir e invertir.

Si este es un horizonte común, entonces hay que conectar los esfuerzos, enlazar las diferencias, y llegar a acuerdos aunque sean mínimos, e investigar metodologías alternativas en busca de la salud global.

Ahora bien, todo esto sería utópico si no sabemos como difundir el mensaje y dar a entender a la ciudadanía, a las empresas y a las instituciones que no se trata de un recorte de su patrimonio personal, comercial, y local, sino de bioética intercultural. Hay unas premisas y convicciones de partida sin las cuales no se puede conseguir el compromiso necesario para crear, asentar y difundir la idea de salud global, para que toda esas propuestas de redistribución de conocimientos médicos, bienes farmacéuticos y servicios sanitarios puedan fluir y organizarse de una manera adecuada y habitual.

Las desigualdades en salud global nos ponen en riesgo por igual a todos. ¿Será el riesgo, a escala global, el que nos obligue a cambiar de mentalidad y pensar la salud en estos otros términos? ¿Será lamentablemente el miedo al cruce entre sida e inmigración lo que produzca alarma y lleve a tomar medidas de prevención o de restricción de acceso? El miedo no es nunca un buen consejero para entender la seguridad, de manera que la primera lección es aprender que lo que nos pone en riesgo por igual son las desigualdades.

Hasta ahora las naciones ricas no habían considerado la salud globalmente, pero cuando se convierte en un problema de seguridad del propio sistema, entonces empiezan a surgir voces, agencias, instituciones y forums que se apuntan a la teoría del riesgo y despliegan iniciativas y objetivos para desarrollos contra el Hambre y la Pobreza. Sin duda los fondos, los discursos y los compromisos en y ante las grandes instituciones son clave, pero también desarrollar el aprendizaje cívico de valores que van a permitir pensar y creer en un proyecto de salud global.

Pero, además, una bioética intercultural para la salud global implica responsabilidad pública y políticas entendidas como ética 
en acción. Esto requiere abrir nuevas vías pedagógicas de co-responsabilidad para que las sociedades más pobres pudieran competir adecuadamente fuera del lastre de los subsidios y ayudas que no permiten implicarse de forma independiente en el desarrollo comercial y en la gestión sanitaria. Y todo ello sobre la base de la solvencia mutua y con auditorias en colaboración.

Un escenario de propuestas que concluye con el requerimiento de constituir una metodología de trabajo unitaria sobre la base de un enfoque interdisciplinario e intercultural: la Bioética Médica, la Ética Tecnocientífica, las Políticas del Desarrollo, y, en especial, la Bioética Intercultural.

Una bioética intercultural que se orienta a trabajar desde la diversidad situacional y contextual como punto de partida para consolidar la discusión, la negociación y los acuerdos entre comunidades tecnocientíficas, culturales y morales. Por lo tanto, no busca exponer principios ni normas de buena actuación entre ciencia, medicina y sociedad, ni vale dejarse llevar por categorías predefinidas y burocratizadas que piensan por nosotros, ni imágenes de la humanidad abstraídas de su historicidad y de relaciones sociales concretas que se activan como esquemas explicativos.

Se trata de diseñar marcos que permitan conectar diferentes realidades sociales y culturales. Y así establecer comunidades de coparticipación intercultural donde discutir diferencias de criterio, animar el flujo de opiniones, razonamientos y propuestas y conseguir decisiones negociadas y concertadas sobre las formas de entender la enfermedad y valorar la salud, el cuerpo integral y fragmentario, las aplicaciones biotecnológicas, las prácticas médicas, entre otras.

Si cabe decirlo con otras palabras aprender a desarrollar un consenso informado sobre lo que se entiende por salud y calidad de vida y, a la vez, establecer los parámetros de la aceptabilidad y la redistribución de los riesgos en contextos culturalmente plurales en sus formas de mantener y restablecer la salud, y, sobretodo, de dar sentido a la solidaridad que pueda ayudar a soportar aquello que de otro modo es insoportable: la conjunción de la enfermedad y la pobreza.

\section{Hacia una tercera cultura \\ A. Pallarés Martí. \\ EUIF Blanquerna. Universidad Ramon Llull. Barcelona}

El Estado Español se ve hoy atravesado por dinámicas contrapuestas. De una parte la integración europea avanzando en distintos ámbitos que reinscriben a sus habitantes en un marco ampliado de pertenencia, marcado por referentes simbólicos tan potentes como la moneda, la residencia jurídica y el derecho al trabajo. Pero al mismo tiempo las migraciones extracomunitarias y su impacto sobre una sociedad golpeada por un cierto desempleo, que en los últimos diez años han cambiado la geografía humana de nuestras ciudades y evidentemente la de nuestras aulas de primaria y secundaria.

Su repercusión en las políticas educativas públicas comenzó a percibirse con la Ley de Reforma Educativa, actualmente generalizada en todas las Comunidades Autónomas. La ley pretende como pilar básico la atención a la diversidad, incluida la cultural, formulando unos complejos contenidos transversales en el currículum escolar formal en torno a temas de interculturalidad. Junto a este bloque, el discurso sobre la identidad, a veces adulteradas y casi siempre contradictorias en torno a las diferencias culturales.

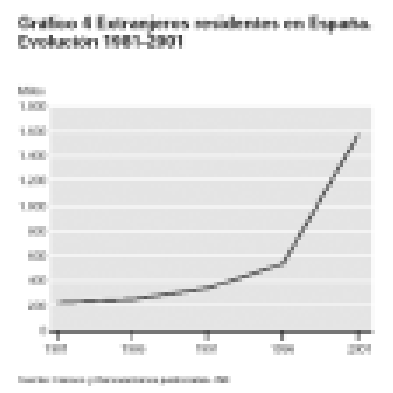

Todavía en clave de la enseñanza obligatoria tenemos que la creciente escolarización de alumnado extracomunitario en los centros educativos de nuestro entorno ha generado tensiones $\mathrm{y}$ desacuerdos entre los diversos agentes sociales, las instituciones y los propios miembros de la comunidad escolar, reabriendo viejos dilemas en torno a cuestiones de multiculturalidad y educación. La nueva composición sociocultural ha servido, en ocasiones, para mostrar las desigualdades ya existentes que se escondían tras un débil halo de homogeneidad cultural, compartida tan sólo en el imaginario colectivo.

El peso de los alumnos hijos de emigrantes extracomunitarios en nuestras aulas es cada vez mayor representando para la ciudad de Barcelona para el curso 2000-01 el 2.86\% del total y en el curso siguiente el $4.35 \%$

\section{Evolución alumnos}

Por nacionalidad según tipo de enseñanza y sector. 2001-2003

\begin{tabular}{lr|r|r|} 
& TOTAL & $\begin{array}{r}\text { Alumnos } \\
\text { Españoles }\end{array}$ & $\begin{array}{r}\text { Alumnos } \\
\text { Extranjeros }\end{array}$ \\
\hline Curso 2000-2001 & 227.830 & 221.312 & 6.518 \\
Curso 2001-2002 & 226.576 & 216.708 & 9.868 \\
Curso 2002-2003 & 227.994 & 213.637 & 14.357 \\
\hline Sector & & & \\
Público & & & \\
Privado & 145.265 & 141.383 & 3.882 \\
\hline & & & \\
Enseñanza & & & \\
Educación infantil & 50.696 & 47.527 & 3.169 \\
Educación primaria & 3.628 & 68.137 & 5.491 \\
Educación especial & 2.007 & 1.897 & 110 \\
Educación secundaria & 101.663 & 96.076 & 5.587
\end{tabular}

Nota: No incluye los alumnos matriculados en las escuelas privadas extranjeras, puesto que no se rigen por la LOGSE. Departament d'Estadística. Ajuntament de Barcelona.

Fuente: Departament d'Ensenyament. Generalitat de Catalunya.

Cualquier aproximación al conocimiento de la realidad social es siempre compleja y, por ello, necesariamente limitada. Esta declaración de principios vale para cualquier fenómeno social y, en particular, para los que se producen en el entorno próximo a la educación la antropología de la educación, ha de saber utilizar las herramientas adecuadas en cada situación para encuadrar y contextualizar los fenómenos que aspira a comprender y explicar, sin 
perder de vista su naturaleza compleja. Como estrategia indagatoria, la antropología no puede, ni debe, desechar los múltiples datos que le informan acerca de su objeto de conocimiento; ya sean datos con los que medir y cuantificar, o datos con los que comprender, interpretándolos, los fenómenos sometidos a estudio.

Los niños y niñas hijos de emigrantes extracomunitarios escolarizados en el Estado español a la vuelta de muy pocos años llegaran de modo natural a la universidad conformando un colectivo muy heterogéneo ofreciendo unas nuevas visiones, desde nuestra perspectiva occidental, que enriquecerán la visión que del mundo de la Salud se percibe en nuestro entorno absolutamente homogéneo. Unas visiones más cercanas a la cosmovisión de una parte importante de ciudadanos en la parte positiva, siempre que este bien gestionada la diversidad.

Esta nueva realidad nos acercara a una realidad que complejiza de inmediato las relaciones entre el colectivo autóctono - profesionales de la Salud, y los "nuevos estudiantes" en su carácter absolutamente heterogéneo, con conceptos sobre el binomio salud/enfermedad diversos, en ocasiones contradictorios entre sí, que ayudaran a ambos colectivos a comprender cómo las personas perciben su mundo y le dan un significado.

Como propuestas final se debe de tender en nuestros entornos tender hacia un multiculturalismo proactivo como una fuerza histórica positiva capaz de enriquecer el imaginario pluralistademocrático, avanzar hacia mayor igualdad de oportunidades y al mismo tiempo hacia mayor espacio para la afirmación de la diferencia.

Un multiculturalismo proactivo necesita conciliar la no-discriminación en el campo cultural con el reparto social frente a las desigualdades. Esto incluye a su vez políticas de acción positiva frente a minorías étnicas, y también frente a otros grupos definidos por estrato socioeconómico, identidad cultural, edad, género o proveniencia territorial.

Sin duda, la migración se ha convertido en uno de los mayores retos de esta centuria.

\section{Emigrar en situación extrema: el síndrome del inmigrante con estrés crónico y múltiple (Síndrome de Ulises). J. Achotegui Loizate}

Si bien las migraciones han sido fenómenos frecuentes en la historia la humanidad, cada migración suele poseer características específicas. Y las circunstancias en las que están llegando actualmente muchos inmigrantes a España y a Europa se caracterizan por ser particularmente difíciles. Emigrar se está convirtiendo hoy para millones de personas en un proceso que posee unos niveles de estrés tan intensos que llegan a superar la capacidad de adaptación de los seres humanos. Estas personas son las candidatas a padecer el Síndrome del Inmigrante con Estrés Crónico y Múltiple o Síndrome de Ulises (haciendo referencia al héroe griego que padeció innumerables adversidades y peligros lejos de sus seres queridos). Se trata de un cuadro clínico que constituye hoy un problema de salud emergente en nuestras sociedades y que surge en el contexto actual de la globalización en el que las condiciones de vida de gran parte de los inmigrantes han empeorado notablemente. Ante esta nueva problemática consideramos que no podemos mirar hacia otro lado y creemos que es nuestro deber darla a conocer a la opinión pública para su debate y resolución.
El Síndrome del Inmigrante con Estrés Crónico y Múltiple se caracteriza, por un lado, porque la persona padece unos determinados estresores o duelos y, por otro lado, porque aparecen una serie de síntomas psiquiátricos que abarcarían varias áreas de la psicopatología. Los estresores más importantes son:

-en primer lugar la soledad y la separación forzada de los seres queridos, especialmente cuando se dejan atrás cónyuges o hijos pequeños a los que no puede traer consigo, ni volver a visitar por problemas de papeles que impedirían posteriormente el retorno al país de acogida

-en segundo lugar el sentimiento de desesperanza y fracaso que surge cuando el inmigrante, a pesar de la enorme inversión que ha realizado en la migración (económica, emocional, etc), no logra ni siquiera las mínimas condiciones para salir adelante al tener dificultades de acceso a "los papeles", al mercado de trabajo, o hacerlo en condiciones de explotación.

-en tercer lugar a todo esto se añade una situación de lucha por la mera supervivencia : dónde alimentarse, dónde encontrar un techo para dormir (al no poder conseguir vivienda por su situación de ilegalidad son víctimas de innumerables abusos).

- y en cuarto lugar el duelo por los peligros físicos del viaje migratorio (las pateras, esconderse en los camiones, etc), o las coacciones ligadas a llegar en viajes "excesivamente organizados" por mafias y grupos que extorsionan y amenazan. Se sabe que el miedo físico es mucho más desestructurante a nivel psicopatológico que el miedo psicológico porque tiene menos posibilidades de salida. También sabemos que el estrés crónico incrementa el condicionamiento del miedo, tanto sensorial como conceptual . Además, en todos los casos existe el miedo a la detención y expulsión.

Esta combinación de soledad, fracaso en el logro de los objetivos, vivencia de carencias extremas, y terror serían la base psicológica y psicosocial del Síndrome del Inmigrante con Estrés Crónico y Multiple (Síndrome de Ulises).

Pero además, el efecto nocivo de las adversidades y peligros que padecen estos inmigrantes se incrementa enormemente por toda una serie de características desfavorables asociadas a las situaciones de estrés:

-el que los estresores sean múltiples, (a más número de adversidades y peligros, mayor riesgo para la salud mental),

-la cronicidad. Estas situaciones límite pueden afectar al inmigrante durante meses o incluso años.

-el sentimiento de que el individuo haga lo que haga no puede modificar su situación (indefensión aprendida),

-el que los estresores posean gran intensidad y relevancia (no es precisamente el estrés de un atasco de tráfico o de unos exámenes)

-el que se asocien a un fuerte déficit de la red de apoyo social,

-el que los propios síntomas (tristeza, fatiga, insominio,..) se conviertan en un nuevo handicap que les dificulta salir adelante.

-a todo esto se han de añadir los duelos clásicos de la migración (ante la nueva lengua, cultura, paisaje...), Duelos a los que hay que añadir ahora la gravedad de los estresores actuales

-y para mayor desgracia para estas personas, el sistema sanitario no atiende adecuadamente muchas veces a estos pacientes:

-bien porque hay profesionales que banalizan esta problemática ( por desconocimiento, por insensibilidad, por prejucios hacia los inmigrantes...)

- bien porque no se diagnostica adecuadamente este cuadro y se trata a estos inmigrantes como depresivos, psicóticos, aña- 
diendo más estresores al inmigrante (aparte del gasto que supone).. Tampoco sus somatizaciones son entendidas como problemas psicológicos y son sometidos a toda una serie de pruebas (como colonoscopias, biopsias, etc...) y tratamientos inadecuados

La expresión clínica del Síndrome del Inmigrante con Estrés Crónico y múltiple es una combinación específica de síntomas: en el área depresiva fundamentalmente tristeza y llanto, pero faltan síntomas básicos de la depresión como apatía, baja autoestima, culpa, pensamientos de muerte (por lo que no se trata de un trastorno depresivo standard), hay también síntomas del área de la ansiedad (tensión, insomnio, pensamientos recurrentes e intrusivos, irritabilidad), síntomas de somatización (cefalea, fatiga, molestias osteoarticulares...), síntomas confusionales (desorientación temporoespacial, despersonalización, desrealización etc). A esta sintomatología se le añade en bastantes casos una interpretación de su cuadro basado en la propia cultura del sujeto. Así es frecuente oir decir: no puede ser que me vayan tan mal las cosas, que tenga tan mala suerte, a mí me han tenido que echar el mal de ojo, me han hecho brujería....

En relación a la denominación de Síndrome se ha de señalar que es meramente descriptiva y que proviene de su acepción más simple: conjunto de síntomas. Y es obvio que estos inmigrantes presentan un buen número de ellos (más de 10 en algunos casos), tal como ya he señalado. De todos modos hay quien prefiere denominar a este cuadro "Mal de Ulises", "Acongojamiento de Ulises"...pero considero que sin tener que recurrir a señalar que estas personas se hallan bien lejos de la definición clásica de la OMS que entiende la salud como "estado de bienestar físico, mental y social”, estos inmigrantes en situación extrema crónica tienen un amplio conjunto de síntomas que se encuadran claramente en la denominación de Síndrome. Sin embargo, ante el reconocimiento de los problemas psicológicos de los inmigrantes ocurre algo muy parecido a lo que ocurre con los padecimientos de la mujer, o de las minorías: se tiende a tener una visión prejuiciada y desvalorizadora de esta sintomatología. desde ciertos planteamientos de la psiquiatría que carecen de sensibilidad ante estas realidades sociales. Tal como escribía hace ya 40 años Frantz Fanon "un negro no es un hombre, un negro es un hombre negro"

Postulamos que existe una relación directa, causal, entre el enorme estrés que viven estos inmigrantes y su sintomatología. Consideramos que las enormes dificultades que viven estos inmigrantes van más allá del típico estrés aculturativo de los inmigrantes (en relación a la lengua, la cultura....).

Soledad, miedo, desesperanza...las migraciones del nuevo milenio que comienza nos recuerdan cada vez más los viejos textos de Homero “...y Ulises pasábase los días sentado en las rocas, a la orilla del mar, consumiéndose a fuerza de llanto, suspiros y penas, fijando sus ojos en el mar estéril, llorando incansablemente...(Odisea, Canto V) ó aquel otro texto en el que Ulises le dice a Polifemo: "Me preguntas cuál es mi nombre. Voy a decírtelo. Mi nombre es nadie y nadie me llaman todos". (Odisea. Canto IX). Es obvio que si un ser humano ha de convertirse en nadie para sobrevivir, ha de ser permanentemente invisible, no poseerá identidad, ni integración social y tampoco puede poseer salud mental.

Malos tiempos aquellos en los que la gente corriente ha de comportarse como héroes para sobrevivir. Ulises era un semidios, que sin embargo, a duras penas sobrevivió a las terribles adver- sidades y peligros a los que se vió sometido, pero las gentes que llegan hoy a nuestras fronteras tan sólo son personas de carne y hueso que sin embargo viven episodios tan o más dramáticos que los descritos en la Odisea.

\section{BIBLIOGRAFÍA}

1. Achotegui, J. 2000. Los duelos de la migración: una perspectiva psicopatológica y psicosocial. En Medicina y cultura. E. Perdiguero y J.M. Comelles (comp). Pag 88-100. Editorial Bellaterra. Barcelona

2. Achotegui, J. 2002. La depresión en los inmigrantes. Una perspectiva transcultural. Editorial Mayo. Barcelona

3. Achotegui. J. 2002. Trastornos afectivos en los inmigrantes: la influencia de los factores culturales. Suplemento Temas candentes. Jano. Barcelona

4. Achotegui, J. 2003 (Comp.). Ansiedad y depresión en los inmigrantes. Editorial Mayo. Barcelona

5. Achotegui, J. 2003 (compilador). Dossier de la reunión internacional sobre el Síndrome de Ulises celebrada en Bruselas en la sede del Parlamento Europeo el 5 de Noviembre del 2003.

6. Achotegui, J. 2004 (compilador). Dossier del Diálogo sobre el Síndrome de Ulises del Congreso "Movimientos humanos y migración” del Foro Mundial de las culturas. Barcelona 2-5 de Septiembre del 2004

7. Achotegui J. 2004. Emigrar en situación extrema. El Síndrome del Inmigrante con estrés crónico y múltiple. Síndrome de Ulises. Norte. Revista de Salud Mental de la Sociedad Española de Neuropsiquiatría. Volumen V No 21. pag 39-53

8. Aguilar, J. 2003. Comunicación personal Sesión clínica Hospital de Sant Pere Claver. Barcelona

9. Beiser, M. 1996. Adjustment Disorder in DSM-IV: Cultural Considerations. In "Culture and Psychiatric Diagnosis. A DSM_IV perspective. Edited by J. Mezzich, A. Kleimman. American Psychiatric Press. Inc. Washington. USA

10. Bilbeny . 2002. Per una ética intercultural. Editorial mediterránea. Barcelona

11. Bon D. 1998. L`animisme. Edtions de Vechi. París

12. Calvo, Francisco. 1970 Qué es ser inmigrante. Barcelona

13. Domic, Z. 2004 Emigrar y enfermar: el Síndrome de Ulises. Congreso Movimientos humanos y migración. Foro Mundial de las Culturas.

14. D`Ardenne P, Mahtani A. 1999. Transcultural counseling in action. Sage publications. London

15. Devereux G. 1951. Psychotérapie d`un indien des plaines. Fayard. París

16. Eliade M. 1972. El mito del eterno retorno. Alianza editorial. Madrid

17. Eliade M. 1977. Ocultismo, brujería y modas culturales. Paidós Buenos Aires

18. Fannon, F. 1970.Escucha blanco. Nova Terra

19. Fernando, S. (Edit. ) 1995. Mental Health in a Multi-ethnic Society. Routledge. London and New York

20. Freud, S. 1912. Tótem y Tabú. Alianza Editorial. Madrid

21. Freud, S. 1930. El malestar en la cultura. Alianza Editorial. Madrid

22. Gómez Mango 2003. Comunicación personal. Colloque Internacional. París

23. Grinberg, L y R. 1994Psicoanálisis de la migración y el exilio. Alianza editorial. Madrid. 
24. Guerrraoui Z, Troadec B. 2000. Psychologie interculturelle. Armand Colin. París

25. Hofstede G. 1999. Culturas y organizaciones. Alianza editorial. Madrid

26. Kareen J, Littlewood R. 1992. Intercultural Therapy. Blackell Science. Oxford

27. Klein. M. 1957. Envidia y gratitud. Paidós. Barcelona

28. J Jenkins R and Üstün B. 1998. Preventing Mental Illnes. Wiley. New York

29. Milne D. 1999. Social Therapy. Willey. New York.

30. Nathan, T. 1999. Médecins et sorciers. Les Empêcheurs de penser en rond. París

31. López-Cabanas M, Chacón F. 1999. Intervención psicosocial y servicios sociales. Síntesis psicología. Madrid

32. Luong Cân Liêm. 2004. De la psychologie Asiatique. L'Harmattan. París

33. Ortigues M. C. y E. Edipo africano. 1974. Tiresias. Ediciones Noé. París

34. Páez D, Casullo M. (comp.) 2000. Cultura y alexitimia. Paidós. Barcelona

35. Roheim G. 1982. Magia y esquizofrenia. Paidós. Buenos Aires

36. Sendi, C. 2001. Estrés, memoria y trastornos asociados. Ariel. Madrid

37. Sow I. 1978. La folie en Afrique Noire. Payot. París.

38. Tizón J, Salamero M, Sanjosé J, Pellejero N, Achotegui J, Sainz F, 1993. Migraciones y salud mental. PPU. Barcelona.

39. Vander Zanden, J.W.1994 Manual de psicología social. Paidós Básica. Barcelona

\section{Atención a la diversidad en salud sexual y reproductiva Carme Ollé Rodríguez}

Ginecóloga SASSIR Raval Sud, Hospital del Mar. IMAS

y Técnica del Pla Director d'Immigració i Cooperació.

Direcció General de Planificació i Avaluació, Direcció

Estratègia i Coordinació, Departament de Salut.

Generalitat de Catalunya.

El aumento de la población inmigrada en nuestro entorno tiene una proyección relacionada de forma muy directa con la salud sexual y reproductiva. En estos últimos años en Cataluña se ha observado y se sigue observando una tendencia ascendente del número de embarazos, muchos de ellos de mujeres nacidas fuera de España, éstas en su mayoría proceden de los países catalogados como de "baja renta". Esta situación se traduce en un incremento de la natalidad, pero tambien en un aumento de las interrupciones voluntarias del embarazo.

Es necesario considerar de forma especial a las mujeres inmigradas y en relación a ellas sus condiciones sociales, económicas y laborales, muy a menudo precarias, todo ello favorece su vulnerabilidad, en especial en relación a la salud afectivo-sexual y reproductiva.

La llegada de la población inmigrada se ha realizado de forma inesperada y brusca en estos últimos años y desde procedencias muy diversas, tal vez por ello su impacto no ha permitido un tiempo de acomodación y conocimiento mutuo en un contexto intercultural.

También es importante recordar:

- Que en la mayoría de los casos la salud no ha sido el motivo para realizar el proyecto migratorio y que han sido otras las prioridades, todas ellas antes que la salud.

- Que la necesidad de acceder o utilizar los servicios de salud a menudo se manifiesta en situaciones consideradas agudas, un ejemplo en las mujeres seria la sospecha o confirmación de un embarazo.

- Que la concepción del sistema de salud de la población inmigrada viene dada por el contexto del país de origen y ésta puede ser muy diferente a la nuestra.

- Que los conceptos de salud al entorno del síntoma, la enfermedad y su tratamiento pueden ser muy diversos y por lo general hay una gran dificultad para la comprensión del concepto "prevención"

Las desigualdades y la vulnerabilidad de esta población en general, tiene una relación directa con la ausencia o el déficit de derechos, la fragilidad o ausencia del soporte social y familiar, el entorno socioeconómico, el incumplimiento de las expectativas y con el propio proceso de duelo migratorio. Todo ello a menudo conlleva a que estas personas y en especial las mujeres presenten un mayor malestar psicológico, tendencia a las somatizaciones y comportamientos de riesgo relacionados con la salud sexual y reproductiva.

El miedo, la desconfianza y la soledad son sentimientos que con frecuencia se presentan en la población que ha inmigrado y pueden relacionarse con la dificultad que presentan para acceder y utilizar los servicios de salud en un contexto de normalidad.

En la atención a la salud de la población inmigrada se tendrían que considerar algunas diferencias específicas de los diversos colectivos según su origen y procedencia. Algunos que se podrían destacar serian: el idioma, la manera de expresarse, los estilos de vida, la religión, los hábitos culturales, los códigos de salud, los patrones familiares y también las circunstancias que les acompañan como las condiciones económicas y laborales, el entorno familiar y social, la vivienda, la dificultad para acceder a los recursos preventivos y el desconocimiento del sistema sanitario local.

En relación al desconocimiento de nuestro sistema sanitario es importante prestar atención a algunas preguntas que a menudo se formula esta población y que tal vez no se ha prestado suficiente atención a dar respuesta.

Algunas de ellas podrían ser:

- Como funciona?

- Como entrar?

- Papeles?

- Tipo de visita: Programada, urgente?

- Centros: Atención primaria, Hospital?

- Gratuidad: Visitas, pruebas complementarias?

Hasta que punto les hemos dado la oportunidad de conocer como funcionamos y el porque?

La perspectiva de género es importante considerarla, la mujer puede ser más vulnerable en relación a la salud afectivo-sexual y reproductiva en consecuencia a las relaciones del poder dominante que se puede establecer desde el hombre y que también pueden influir en una mayor expresión de la violencia de género.

El rol de la maternidad en el país de origen a menudo esta directamente relacionado con el concepto de riqueza, continuidad y futuro, esto puede favorecer en esta población la tendencia a la multiparidad y a los intervalos inter - genésicos cortos.

También hay que considerar el embarazo en la adolescencia, los embarazos no deseados y en consecuencia la demanda de inte- 
rrupción del embarazo, así como la dificultad para acceder a una contracepción adecuada y las conductas de riesgo que favorecen las infecciones de transmisión sexual.

Si bien algunos de estos aspectos son comunes a los de las poblaciones desfavorecidas, en relación a las mujeres que han inmigrado, será importante el abordar estas situaciones en un contexto intercultural.

En la atención a la población inmigrada hay que poner en activo un proceso de aproximación mutua y favorecer el vínculo con una actitud acogedora. Es necesario que el profesional observe, escuche, pregunte aquello que desconoce de su cultura. Hay que evitar los estereotipos, garantizar el respeto y comprender sin juzgar. Se ha de conseguir poder dar una información adecuada para así garantizar una asistencia de calidad.

Los movimientos humanos actuales ponen de manifiesto la necesidad de adecuar los recursos a las necesidades, y por ello la importancia de mejorar las habilidades de los profesionales.

Así pues es necesario que se ofrezcan programas de formación específicos en conocimientos, actitudes y comportamientos que permitan atender la diversidad cultural, garantizando la capacitación de los profesionales en competencias interculturales.

Estos programas deberían estar dirigidos no solo a los profesionales de la salud actuales sino también para los futuros profesionales, ellos son el mañana y nuestro mañana sin lugar a dudas será intercultural.

\section{Diversidad cultural y Salud: una propuesta de formación inicial para profesionales de la educación \\ Enric Prats Gil. Facultad de Pedagogía \\ Universidad de Barcelona}

La diversidad cultural ha entrado en las aulas y espacios educativos, formales y no formales, y ha modificado actitudes y así como prácticas de muchos profesionales, a pesar de los vacíos todavía existentes. La interculturalidad se apunta como un horizonte, aún bastante lejos, a partir del cual se diseñan los programas educativos y ahora también la formación inicial de los profesionales de la educación. Aun así, la función que tiene la salud, como base del despliegue de la personalidad y por lo tanto de la educación de las personas, es uno de los contenidos poco desarrollados en estos programas formativos; aún más cuando hablamos de salud internacional, de salud de las personas inmigrantes, de la relación entre salud y cultura; en definitiva, de una perspectiva transcultural de la salud. En esta aportación describiré los rasgos esenciales de la formación inicial de pedagogos y educadores en materia de salud e interculturalidad; en concreto, explicaré los objetivos y contenidos de esta formación así como los instrumentos necesarios para que incrementen su competencia. La propuesta se justifica en la convicción de que la educación, en general, tiene que dar respuesta a las expectativas y necesidades de sus usuarios y que la educación para la salud, en concreto, no puede eludir el análisis y la valoración de las relaciones entre salud y culturas.

Salud y diversidad cultural en los estudios de Pedagogía

Conviene contextualizar con brevedad en qué escenario universitario ubicamos la propuesta que se presenta. Los estudios de Pedagogía tienen la finalidad de formar profesionales que ejecutarán tareas de soporte, asesoramiento, formación, elaboración de material, dirección y aplicación de proyectos educativos en diversos ámbitos, no sólo escolares, sino también sociales, en grandes empresas, etc. En cambio, los estudios de Educación Social forman a educadores que tendrán que llevar a cabo, de manera prioritaria, tareas de intervención educativa en contextos no formales (ocio, prisiones, gente mayor, asociaciones, etc.).

En este contexto, la asignatura de Educación para la Salud (EPS), opcional en Pedagogía y obligatoria en Educación Social, pretende proporcionar a los futuros profesionales los instrumentos imprescindibles de reflexión y actuación en el ámbito de la salud, aspecto que se trabaja tangencialmente en otras asignaturas (Drogas, Discapacitación, etc.) y también en algún practicum. Precisamente, a partir de las prácticas en entornos educativos, los estudiantes se dan cuenta de la diversidad de procedencias y adscripciones de los educandos (alumnos de escuela, usuarios o participantes de escuelas de adultos, etc.), y cuando específicamente les interesa el tema de la salud chocan con muchos prejuicios personales y una falta absoluta de formación.

Así, partimos de la premisa de que los estudiantes tienen un conocimiento escaso del mundo de la salud y que, de entrada, no ven la relación entre educación y salud. De hecho, como el resto de la población, los estudiantes tienen una posición inicial de desinterés y de rechazo hacia los temas de salud, que se refleja en la falta de una posición definida y clara en temas como el afrontar enfermedades graves, la muerte, y también a la hora de dibujar qué es un estilo de vida saludable, más allá de lo que se pueda percibir debido a la presión de los medios de comunicación.

Para resumir, la asignatura de EPS se organiza en tres ejes programáticos:

- competencial, con el objetivo de proporcionar instrumentos de reflexión y de intervención para elaborar proyectos educativos que promuevan estilos de vida saludables y capaciten para detectar y superar situaciones de riesgo;

- conceptual, con el objetivo de incrementar en los estudiantes los conocimientos sobre salud para que sean capaces de superar el concepto de salud como ausencia de enfermedad, o como estado de equilibrio bio-psico-social, y se acerquen más hacia a una noción de salud como derecho fundamental de las personas;

- actitudinal, con el objetivo de fomentar una posición favorable a los temas de salud y despertarlos la inquietud de incorporar la salud como eje programático en cualquier clase de proyectos educativos.

El motor de la propuesta es que la salud tiene que convertirse en un tema de compromiso socioético con la consideración pedagógica que ello significa desde el punto de vista metodológico. Así, y en la línea de las pautas del proceso de convergencia universitaria en Europa, que centra el proceso en el aprendizaje del estudiante, la asignatura de EPS se basa en dos mecanismos: el análisis y la valoración de temáticas sociales destacadas, y la investigación en grupos cooperativos de experiencias de salud.

La construcción de conocimientos en salud y la variabilidad cultural.

De modo muy esquemático, y sólo a efectos didácticos para los estudiantes, proponemos que los humanos construimos nuestros conocimientos y convicciones sobre la salud a partir de una tríada de nociones inseparables: certezas, creencias y gustos. De hecho, acabamos prefiriendo $\mathrm{u}$ optando por una determinada conducta en salud según el peso que en cada momento damos a las nociones en juego. Las fuentes a partir de las cuales se elaboran estos tres tipos de nociones son varias y no cabe desci- 
frarlas aquí, pero nos interesa apuntar su influencia en el tema que nos ocupa.

Las certezas se derivan del contraste empírico y validado por el método científico, cuyos resultados aspiran a tener validez universal; aquí se debería incorporar la experiencia cotidiana de cada uno, cuando confirma las teorías científicas, pero que se convierte en creencia cuando la ciencia no le funciona. Así, las creencias se apoyan en el saber popular, en el conocimiento vulgar, validadas por la tradición y la experiencia personal intransferible y a menudo inexplicable ("a mi me funciona este remedio"); las creencias tienen una adscripción cultural y un fuerte apego en personas que participan activamente del imaginario compartido para la comunidad. Finalmente, los gustos, en tanto que preferencias personales, son elecciones basadas en un variado y difuso repertorio de ingredientes que, aún con la presión del medio social, son responsabilidad única y exclusiva del individuo; a menudo, el gusto sólo se vincula con la estética, pero se deben tener presentes muchas convicciones en salud construidas sobre los binomios placer-riesgo o deber-interés, que muchas veces confundimos con una tensión más general entre emoción y razón, pero que no dejan de ser elecciones por inclinación o propensión, por gustos, en definitiva.

Los tres ámbitos citados corresponden, por lo tanto, a tres niveles concéntricos de construcción del conocimiento que podemos graduar en una escala que va del más universal al más personal, pasando por un estadio intermedio de carácter comunitario o cultural. La tesis que sostenemos es que en una sociedad uniformizada en un mismo código cultural es probable que se produzcan grandes coincidencias entre las certezas y las creencias, y que puede haber un pequeño margen para los gustos individuales. Ahora bien, en una sociedad multiformada con varios códigos culturales, la distancia entre certezas y creencias aumenta y seguramente el margen para los gustos individuales se amplía de manera exponencial. Los procesos de asimilación cultural se encargan de subsumir las creencias culturales de los grupos menos potentes en las creencias del grupo dominante, que según como pueden alcanzar la categoría de certezas. De ahí la importancia del método científico, como instrumento (y quizás sólo como instrumento) para mantener la separación, tan útil, entre certezas y creencias.

Instrumentos para incrementar la competencia en salud: contenidos de EPS.

Una perspectiva transcultural de la salud nos permite obtener un mapa conceptual de contenidos que agrupamos en cuatro bloques temáticos:

- Globalización, movimientos migratorios y salud internacional. Conocimiento de las relaciones que se producen entre la economía, la demografía y la salud pública. El incremento de los flujos de personas (inmigrantes, turistas, cooperantes, deportistas, ejecutivos, etc.) a escala mundial alteran substancialmente los mapas epidemiológicos y los patrones de salud. En otro orden de cosas, el estudiante conoce el poder de la farmaindústria y percibe con facilidad las razones por las cuales se fomentan determinadas líneas de investigación, siendo capaz de analizar y valorar las problemáticas relacionadas con las patentes, las enfermedades de "pobres", las patologías raras, etc., pero no alcanza a vislumbrar el gap que se da en los procesos de transición epidemiológica entre países ricos y países pobres.

- Trastornos de salud directamente relacionados con la cultura, el origen, etc. Derivado del bloque anterior, se trata de identificar la morbididad atribuible a procedencia geográfica, pero también a situaciones sociales imbricadas con creencias culturales. La salud de las personas inmigrantes y, en concreto, el síndrome de Ulises es uno de los ingredientes más trabajados en este ámbito; además, los educadores de adultos informan que este fenómeno está muy presente en bastantes usuarios de sus aulas.

- Medicina alopática y medicinas alternativas o complementarias. En este bloque se trata de abordar la problemática derivada de la diversidad de soluciones o terapias que los diversos grupos sociales y culturales han adoptado en cada momento, y comprobar las tensiones que se producen en los puntos de fricción, como son, por ejemplo, la variabilidad cultural de las pautas de crianza y de salud materno-infantil y el papel del curanderismo, etc. También hay que incidir en el estereotipo que atribuye una función salvadora a la medicina oficial y el papel de los medios de comunicación en la difusión y propagación de creencias y certezas en salud, etc.

ß Acceso a los servicios de salud y distancia cultural, comunicación con los enfermos y con las familias, etc. En este bloque se trata de entrar a estudiar los mecanismos que facilitan una atención sanitaria culturalmente adaptada que reduzca las dificultades de acceso a servicios de salud por parte de determinados colectivos. De hecho, el núcleo central será el análisis de las condiciones que permiten una relación fluida entre profesionales y agentes de salud con los usuarios, y las habilidades comunicativas que facilitan diagnósticos ajustados y que aumentan el mantenimiento de las prescripciones médicas.

En resumen, los estudiantes tienen que ser capaces de fomentar determinadas competencias en los usuarios de los servicios educativos, tanto niños como adultos, que concentramos en tres apartados:

a) Competencia emocional y perceptiva, que incluye el reconocimiento de la variabilidad cultural de las emociones (gestión cultural de las emociones), el reconocimiento de la variabilidad de las percepciones sobre la gravedad y la vulnerabilidad en salud, y el reconocimiento del propio cuerpo, en el sentido de aprender a adoptar distancia emocional con uno mismo. En escuelas con niñas inmigrantes, se han producido situaciones puntuales de (auto)discriminación a la hora de hacer educación física, apelando a convicciones religiosas o pautas culturales; una intervención educativa sobre las emociones y las percepciones sobre el propio cuerpo se hace inexcusable en estos casos.

b) Competencia ético-cognitiva, que incluye capacidades de autoconocimiento, de autoanálisis y autoexploración (para reconocer e interpretar signos y síntomas), conocimientos sobre la realidad y comprensión crítica del entorno (dificultades en el acceso a servicios sociosanitarios, por ejemplo, y también el reconocimiento de la relación entre desigualdades socioeconómicas y déficits sociosanitarios; lectura crítica de los medios audiovisuales como reproductores de arquetipos monoculturales de salud, etc.), y las capacidades de juicio y razonamiento, como instrumentos para reflexionar y poner en discusión permanente las creencias sobre la salud y los principios morales asociados al cuerpo.

c) Competencia social, que incluye capacidades de autorregulación (como capacidades de resistir la presión del grupo, y relacionada con la toma de decisiones sobre hábitos, conductas y estilos de vida), las habilidades sociales y habilidades para el diálogo (facilitadoras de la comunicación intercultural, de las relaciones con personal sanitario y de las relaciones con otros enfermos, 
etc.), la empatía y las manifestaciones culturales de la empatía; y las capacidades para adaptarse al entorno $y$, si es necesario, modificarlo, que se traducen en destrezas proyectivas, de organización y planificación, etc.

Conclusiones: Una perspectiva de promoción de la salud tiene que revisar la relación cuerpo y salud; si bien el cuerpo corresponde al ámbito de la responsabilidad individual, la salud entra en el de la responsabilidad compartida por la comunidad, en una relación dialéctica y simétrica. La tríada formada por ciencia, cultura y persona tiene que intentar constituirse y mantenerse como un triángulo equilátero, con equidistancias ajustadas, sin que ningún ángulo domine sobre los otros dos. Es relativamente sencillo apuntar los riesgos derivados de desequilibrar éste triángulo. Determinadas "ofertas" culturales pueden tener la tentación de intervenir en el espacio reservado a la individualidad, al cuerpo mental y físico en definitiva, pero al mismo tiempo corremos el riego de "caer" en manos de la ciencia mediática todopoderosa, que pretenderá anular las derivas culturalistas.

Es importante, insistimos, que los programas educativos de cualquier clase, tanto los escolares como los del ámbito comunitario, recojan la necesidad de incidir en una perspectiva transcultural de la salud en la línea señalada en esta aportación, y para conseguirlo, la formación inicial en Educación para la Salud dirigida a profesionales de la educación es uno de los instrumentos más destacados. 